\title{
The effects of magnesium sulfate therapy after severe diffuse axonal injury
}

\author{
This article was published in the following Dove Press journal: \\ Therapeutics and Clinical Risk Management \\ 27 September 2016 \\ Number of times this article has been viewed
}

\author{
Ling Zhao' \\ Wei Wang' \\ Jiwen Zhong' \\ YaYun $\mathrm{Li}^{\prime}$ \\ YanZi Cheng' \\ Zhenjiao Su' \\ Wei Zheng' \\ Xiang-Dong Guan ${ }^{2}$ \\ 'Department of Critical Care \\ Medicine, Zhuhai People's Hospital, \\ Zhuhai, Guangdong, ${ }^{2}$ Department \\ of Critical Care Medicine, The First \\ Affiliated Hospital of Sun Yat-sen \\ University, Guangzhou, Guangdong, \\ People's Republic of China
}

Correspondence: Xiang-Dong Guan Department of Critical Care Medicine, The First Affiliated Hospital of Sun Yatsen University, No 58, the Second Road of ZhongShan Road, 239000, Guangzhou, Guangdong, People's Republic of China

Tel +86 $208733 \quad 1008$

Fax +86 2087331008

Email 1372702105I@sina.cn
Purpose: To evaluate the clinical effects of magnesium sulfate in the treatment of diffuse axonal injury (DAI).

Patients and methods: This study was a randomized, double-blind, placebo-controlled trial conducted in the First Affiliated Hospital of Sun Yat-sen University, Guangzhou and Zhuhai People's Hospital, Zhuhai, two trauma center hospitals. A total of 128 patients suffered from DAI, with initial Glasgow coma scale (GCS) scores of 3-8. They were randomly divided into two groups: magnesium sulfate treatment (MST) group ( $n=64)$ and control group $(n=64)$. The MST group received $250 \mu \mathrm{mol} / \mathrm{kg}$ magnesium sulfate intravenously 20 minutes after admission, followed by $750 \mu \mathrm{mol} / \mathrm{kg}$ magnesium sulfate intravenously daily for 5 days. The control group received standard management without MST. GCS scores and serum neuron-specific enolase values were measured and recorded at admission, and on days 3 and 7 after injury. Outcomes were determined by Glasgow outcome scale scores at discharge and at 3 months' follow-up, respectively.

Results: After the 7-day treatment, patients in the MST group, compared with those in the control group, had a lower serum neuron-specific enolase level (25.40 \pm 6.66 vs $29.58 \pm 7.32$, respectively, $P=0.001)$ and higher GCS score $(8.23 \pm 2.72$ vs $7.05 \pm 2.64$, respectively, $P=0.016)$. Although the length of stay and mortality did not differ between the groups in the intensive care unit, Glasgow outcome scale score was significantly lower in the MST group at discharge (3.30 \pm 1.35 vs $3.90 \pm 1.10, P=0.004$ ) and 3 months after discharge ( $2.95 \pm 1.48$ vs $3.66 \pm 1.44, P=0.009$ ).

Conclusion: Early treatment with magnesium sulfate resulted in a significant improvement in DAI outcome. Further studies are needed to confirm the clinical significance of treatment of DAI patients with magnesium sulfate.

Keywords: diffuse axonal injury, magnesium sulfate, outcome

\section{Introduction}

Diffuse axonal injury (DAI) is one of the most common and severe types of traumatic brain injury (TBI) after vehicle accidents. ${ }^{1}$ When screening all forms of TBI, it has been confirmed that $60 \%$ of all severe and $30 \%$ of moderate brain injury victims are associated with DAI. ${ }^{2}$ It has been proven that severe DAI can cause an increased incidence of morbidity, mortality, and development of a persistent vegetative state. . $^{3,4}$ The severity of injury is typically more in younger than older people but the outcomes are worse in the elderly. ${ }^{5}$

Brain ischemic damage as a result of secondary brain injury is extremely common in the later period following TBI, with $80 \%$ to $90 \%$ of the victims showing evidence of ischemic brain damage by histopathological examinations. ${ }^{6,7}$ Recently, researchers observed that an ultra-early period of severe reduction in brain blood flow occurs within 6 hours after the initial insult in one-third of the patients with severe TBI. ${ }^{7}$ 
This ultra-early ischemia is characterized by focal reductions in cerebral blood flow below the ischemic threshold of $18 \mathrm{~mL} / 100 \mathrm{~g} / \mathrm{min}$, which leads to poor blood supply and ischemic neuronal necrosis. ${ }^{7}$ Heath and Vink have ${ }^{8}$ shown that there is a significant and sustained decrease in free $\mathrm{Mg}^{++}$ concentration in the brain after traumatic brain axonal injury; it is correlated with the neurological deficit and prognosis. Previous research has shown that administration of magnesium sulfate was an effective treatment for focal or diffuse TBI, when given a bolus 30 minutes after injury at least; treatment with magnesium sulfate improved neurological outcome versus treatment with placebo. ${ }^{9,10}$ Hence, we believe it is of high clinical value to further investigate the impact of early and sustained magnesium therapy in TBI. It is wellknown that mild hypothermia may improve brain injury, magnesium as a potential neuroprotective agent, and may have an effect in the treatment of head injury and brain ischemia. The mechanism is more likely to be obtained by an increase in magnesium concentration in plasma and then maintaining magnesium level in brain extracellular fluid. ${ }^{11-13}$ The study was designed to observe the clinical effect of magnesium sulfate in the treatment of DAI. To our knowledge, there are few existing studies that have examined the effect of magnesium sulfate in the treatment of DAI. If magnesium eventually is found to be even moderately effective as a neuroprotective agent in TBI, there may be significant advantages. It is widely available, inexpensive, and has an established safety profile. This contrasts with concerns over the safety of noncompetitive $N$-methyl-D-aspartate (NMDA) antagonists in particular. The purpose of this study was to observe the clinical outcome of early administered magnesium after TBI.

\section{Patients and methods}

\section{Participants}

This study was a randomized, double-blind, placebo-controlled design conducted in two hospitals. We studied a total of 128 patients with DAI at the Department of Critical Care Medicine of Zhuhai People's Hospital and the First Affiliated Hospital of Sun Yat-sen University (two regional trauma centers in People's Republic of China) during a 17-month period between January 1, 2013, and May 31, 2014. The protocol was approved by the Ethics Committee of the Zhuhai People's Hospital and the First Affiliated Hospital of Sun Yat-sen University. Written informed consent was obtained from each patient or one family member before inclusion into the study. The study protocol is outlined in Figure 1.

All patients presenting with a diagnosis of DAI were checked for eligibility by the treating physician. Inclusion

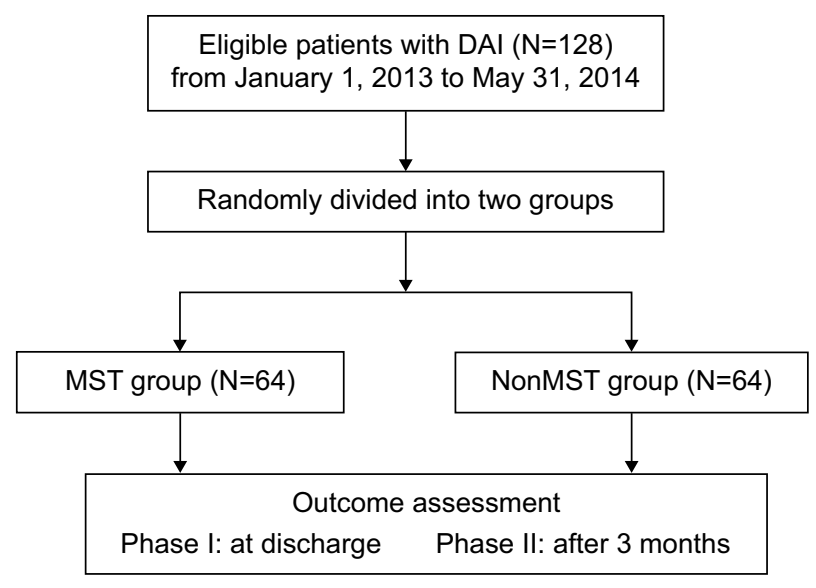

Figure I Flow chart of the study.

Abbreviations: DAI, diffuse axonal injury; MST, magnesium sulfate treatment.

criteria were: 16 to 72 years of age and nonpenetrating head injury. Exclusion criteria were: patients with major associated extracranial injuries, patients who underwent craniotomy, and patients refusing participation. The sample population was composed of 104 males and 24 females, ranging in age from 16 to 72 years (mean age $41.04 \pm 17.15$ years). All patients were admitted directly within 1 hour of the incident. Motor vehicle accidents accounted for $73 \%$; while the remaining injuries were due to the result of falls, assaults, and other causes. Patients were examined by cranial computed tomography or magnetic resonance imaging immediately on admission. Patients with DAI were diagnosed according to Adams and Gennarelli's definition. ${ }^{1,6,14}$ Normal computed tomography findings of small or collapsed ventricles, subarachnoid hemorrhage, intraventricular hemorrhage, or small hematomas in the white matter with no or $<5 \mathrm{~mm}$ midline displacement, or a combination of these, and Glasgow coma scale (GCS) scores of $\leq 8$ at hospitalization, were included in this study. Patients with injury to multiple systems were not admitted to our intensive care units and therefore are not a part of our study.

\section{Randomization}

Patients who met the eligibility criteria were assigned to either the control group (placebo) or magnesium sulfate treatment (MST) group, by stratified block randomization. This was carried out using a computer system managed by a statistician not related to the project team in order to protect the double-blind design and integrity of the study. Randomization was stratified by sex, age (16-72 years of age), and GCS scores of $\leq 8$ at hospitalization. The allocation ratio was $1: 1$. Patients, treating physicians, and investigators 
assessing outcomes and analyzing data would be unaware of the allocation.

\section{Protocol for management and administration of DAI}

Patients were promptly intubated if they manifested any signs of respiratory distress. Patients were electively intubated if their GCS scores were $\leq 6$ or if they displayed poor airway protective reflexes. An arterial line and central venous catheter were routinely placed. Mechanical ventilation was adjusted to keep partial pressure of carbon dioxide in the arterial blood between 32 and $35 \mathrm{mmHg}$. Patients underwent complete neurological assessment by the nursing staff hourly (more frequently if deteriorating). Hyperventilation, mannitol, and cerebral spinal fluid drainage were only used if patients had signs of elevated intracranial pressure $(>20 \mathrm{mmHg})$. The MST group received $250 \mu \mathrm{mol} / \mathrm{kg}$ magnesium sulfate intravenously 20 minutes after admission, followed by $750 \mu \mathrm{mol} / \mathrm{kg}$ magnesium sulfate intravenously daily for 3-5 days. The control group received standard management without MST. The regimen chosen was intended to result in a serum magnesium concentration twice the physiological concentration, and maintain this level for 24 hours.

\section{Data collection}

The following information was collected from the medical records of each patient: age and sex details of initial injury, GCS score recorded at admission, and on days 3 and 7 after the traumatic injury. Serum neuron-specific enolase (NSE) values were measured at admission, and on days 3 and 7 . Outcome was determined by Glasgow outcome scale (GOS) score at discharge and 3 months after discharge for each patient. The GOS scores were defined as: I = mild or no disability, II = moderate disability, III = severe disability, $\mathrm{IV}=$ vegetative state, and $\mathrm{V}=$ death. The length of stay both in the intensive care unit and hospital was recorded.

\section{Statistical analysis}

Data analysis was performed with a statistical software (SPSS for Windows, version 15.0; SPSS Inc., Chicago, IL, USA). All values were presented as mean \pm standard deviation. Mean values and frequencies were compared by using analysis of variance and $x^{2}$ analysis, respectively. We performed stepwise logistic regression analysis to determine the effect of magnesium sulfate on in-hospital mortality after adjusting for age, initial GCS score, and initial serum NSE. Age and initial GCS score were entered in the model. We performed a second stepwise linear regression analysis to evaluate the effect of magnesium sulfate (MS) on GOS at discharge after adjusting for age, initial GCS score, and initial serum NSE. An entry criteria of $P<0.1$ was used in this model. A $P$-value of $<0.05$ was considered significant.

\section{Results}

A total of 128 patients with DAI were treated with and without magnesium sulfate from January 1, 2013, to May 31, 2014, a comparison between the characteristics of the two groups is presented in Table 1. No significant differences were observed between the two groups with regard to age and initial GCS scores. We found significant improvement in the GCS score on days 3 and 7 after admission in the MST group (7.39 \pm 2.07 with MST and 6.23 \pm 2.29 without MST, $P=0.004)$ and (8.23 \pm 2.72 with MST and 7.05 \pm 2.64 without MST, $P=0.016$ ), respectively. Although initial serum NSE was similar in both groups, maximum NSE recorded during the intensive care unit stay was significantly higher in the control group (Table 1). There was no difference in the frequency of hyperventilation, mannitol, and cerebrospinal fluid drainage between the two groups. The in-hospital mortality was lower in the MST group. The outcome at discharge and 3 months after discharge between the two groups is shown in Figures 2 and 3. The mean GOS score was significantly lower in the MST group at dis-

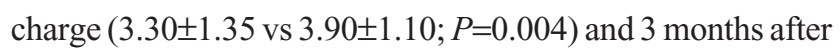

Table I Baseline characteristics and outcome of patients who did or did not receive MST treatment

\begin{tabular}{|c|c|c|c|}
\hline Characteristics & MST & NonMST & $\overline{P \text {-value }}$ \\
\hline $\mathrm{N}$ & 64.00 & 64.00 & \\
\hline Age, years & $39.61 \pm 17.14$ & $41.01 \pm 16.38$ & 0.767 \\
\hline Sex (\% male) & $53.00(82.8 I)$ & $51.00(79.68)$ & 0.325 \\
\hline APACHE II score AD & $21.81 \pm 2.75$ & $21.61 \pm 2.44$ & 0.677 \\
\hline Glucose, mmol/L AD & $\mid 0.60 \pm 1.71$ & $10.25 \pm 1.40$ & 0.208 \\
\hline NSE, g/L AD & $32.76 \pm 6.88$ & $33.12 \pm 6.13$ & 0.756 \\
\hline NSE, $g / L$ after 3 days & $28.93 \pm 6.50$ & $32.11 \pm 7.09$ & 0.100 \\
\hline NSE, g/L after 7 days & $25.40 \pm 6.66$ & $29.58 \pm 7.32$ & 0.001 \\
\hline GCS AD & $5.82 \pm|.7|$ & $5.36 \pm 1.91$ & 0.159 \\
\hline GCS after 3 days & $7.39 \pm 2.07$ & $6.23 \pm 2.29$ & 0.004 \\
\hline GCS after 7 days & $8.23 \pm 2.72$ & $7.05 \pm 2.64$ & 0.016 \\
\hline GOS at discharge & $3.30 \pm 1.35$ & $3.96 \pm 1.10$ & 0.004 \\
\hline GOS after 3 months & $2.95 \pm 1.48$ & $3.66 \pm 1.44$ & 0.009 \\
\hline The events at discharge & $46(70.31)$ & $52(81.25)$ & 0.149 \\
\hline The events after 3 months & $35(54.69)$ & 51 (79.69) & 0.001 \\
\hline ICU duration, days & $21.04 \pm 9.39$ & $23.67 \pm 14.59$ & 0.231 \\
\hline ICU mortality (\%) & $20(31.25)$ & $26(40.62)$ & 0.167 \\
\hline
\end{tabular}

Notes: Events. GOS of patients were III, IV, and V (the poor prognosis group). $P$-value of $<0.05$ was considered as significant.

Abbreviations: AD, admission; APACHE, acute physiologic score and chronic health evaluation; GCS, Glasgow coma scale; GOS, Glasgow outcome scale; ICU, intensive care unit; MST, magnesium sulfate treatment; NSE, neuron-specific enolase. 


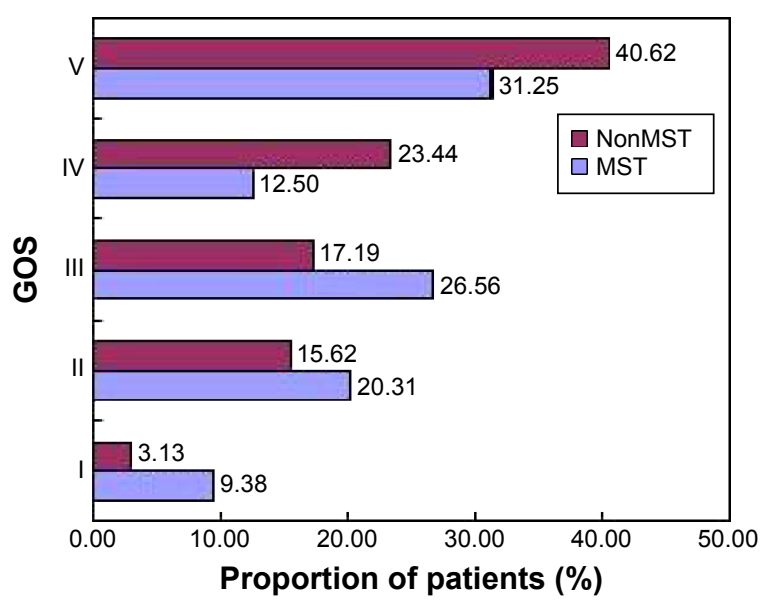

Figure 2 The distribution of the two groups in the different prognosis at discharge. Abbreviations: GOS, Glasgow outcome scale; MST, magnesium sulfate treatment.

discharge ( $2.95 \pm 1.48$ vs $3.66 \pm 1.44 ; P=0.009)$. The cumulative proportion of patients who demonstrated improvement in neurological status (improvement in GCS score of $>2$ points compared to admission score) was higher in the MST group on days 3 and 7. According to GOS: $\mathrm{I}=$ mild or no disability and II = moderate disability were defined as a negative event $=0$ (the good prognosis group); III = severe disability, IV = vegetative state, and V = death were defined as a positive event $=1$ (the poor prognosis group). The survival curves (Figure 4) showed lower survival rate for the MST group but the difference was not statistically significant $(P=0.077)$. Three months after hospital discharge, the cumulative survival rate in the MST group was significantly improved. Figure 5 shows a differentiation in the Kaplan-Meier curve and after the Cox regression analysis, a survival benefit for the MST group $(P=0.018)$. This shows that magnesium does not significantly improve the prognosis of DAI patients in the short term, but may improve long-term prognosis.

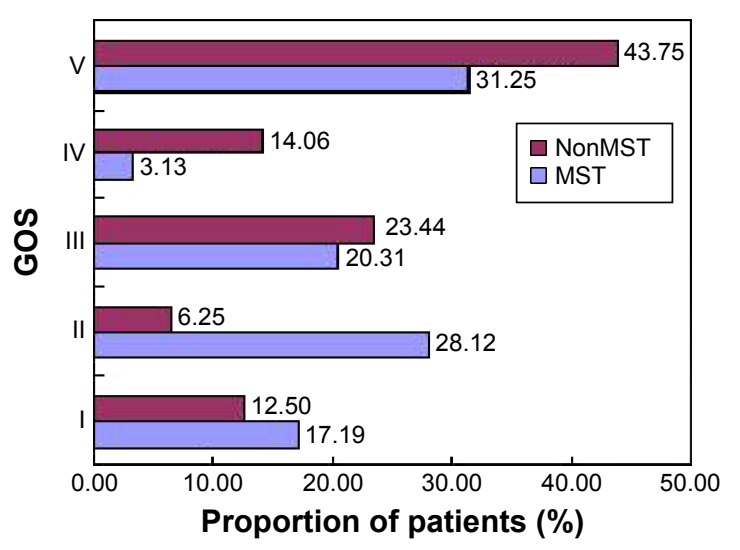

Figure 3 The distribution of the groups in the different prognosis after 3 months. Abbreviations: GOS, Glasgow outcome scale; MST, magnesium sulfate treatment.

\begin{tabular}{|l|l|l|l|l|l|}
\hline & $\boldsymbol{B}$ & $\boldsymbol{P}$ & HR (B) & \multicolumn{2}{|c|}{$\mathbf{9 5 \%}$ Cl for HR (B) } \\
\hline & & & & Lower & Upper \\
\hline Group & 0.389 & 0.077 & 1.476 & 0.959 & 2.273 \\
\hline
\end{tabular}

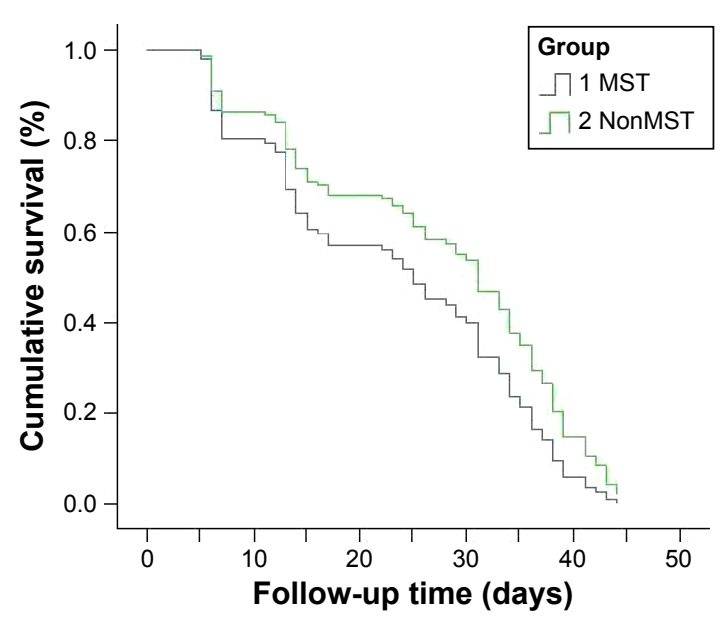

Figure 4 Comparison of the survival between the two groups at discharge. Abbreviations: $\mathrm{Cl}$, confidence interval; MST, magnesium sulfate treatment; $P, P$-value; $H R$, hazard rate ratio; $B$, regression coefficient.

\section{Discussion}

DAI remains the leading cause of morbidity and mortality in major brain injury, which occurs in more than half of all severe cases of clinical TBI. ${ }^{3,4}$ Early histopathological studies using silver staining techniques estimated that nearly $25 \%$ of patients ultimately died from severe TBI. ${ }^{15}$ However, using more sensitive indicators, such as $\beta$-amyloid immunohistochemical analyses, more recent studies indicate that axonal injury has been commonly observed and reactive axonal

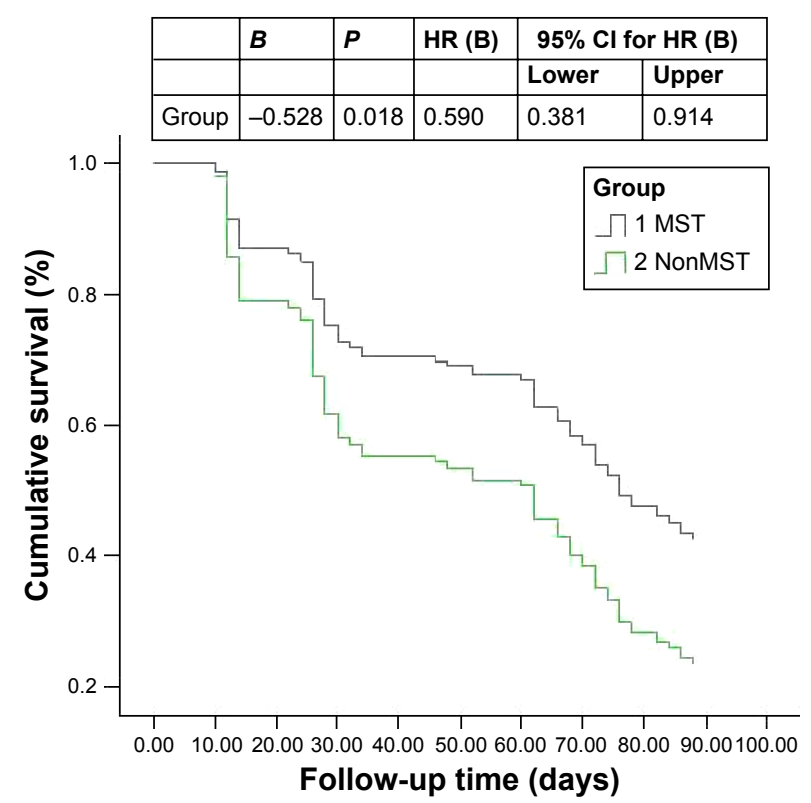

Figure 5 Comparison of the survival between the two groups after 3 months. Abbreviations: $\mathrm{Cl}$, confidence interval; MST, magnesium sulfate treatment; $P, P$-value; $H R$, hazard rate ratio; $B$, regression coefficient. 
changes found in $>90 \%$ of cases in severe TBI. ${ }^{4}$ The cause of axonal injury was believed to be due to injury that occurred as a direct result of the tensile forces of the primary initial trauma, but is now recognized that delayed process of progressive intra-axonal changes leads to disconnection and is considered part of the secondary axonal injury. ${ }^{11,16}$ This delayed process has been shown to be present between hours and days after the traumatic event and is thought to be largely responsible for many of the neurologic deficits in the posttrauma period. ${ }^{15,16}$ The current understanding of the mechanisms of axons injury is dramatically applied to the therapeutic approach. This study presents that the use of magnesium sulfate can improve neurologic impairment and outcome of DAI patient. Figures 2 and 3 show the outcome for the two study groups at discharge and 3 months after discharge. It is clear that the MST group had better outcome than control group.

In general, mechanical disruption of axon injury seldom results from direct TBI. Pathologically, traumatic shearing injury with focal, breaking of the subtle axonal cytoskeleton may lead to impaired axoplasmic transport, continued axonal swelling, and ultimate disconnection. ${ }^{3,15}$ According to the recent research about initial intra-axonal events that are triggered, the described sequence of reactive axonal change is multifactorial, such as axolemmal permeability, direct cytoskeletal damage/perturbation, or more overt metabolic/ functional disturbance. ${ }^{12,15}$ Traumatically induced DAI will lead to diffuse deafferentation, which is a pathobiological change associated with morbidity and mortality. ${ }^{15}$

Magnesium as a neuroprotective agent is approved by many preclinical models of ischemic and excitotoxic brain injury. ${ }^{17-19}$ Even with a severe brain injury, correcting magnesium deficiency is believed to prolong the victim's life. ${ }^{20}$ Compared to a great majority of other neuroprotective agents, there are a number of clinical research studies with magnesium, such as preeclampsia/eclampsia and myocardial infarction, which confirm its safety and tolerability. A number of possible mechanisms of magnesium are considered, including that the $\mathrm{Mg}^{2+}$ ion regulates cellular energy metabolism, vascular tone, and cell membrane ion transport. Magnesium is used generally to antagonize calcium in plasma. Furthermore, adenosine triphosphate concentrations may be regulated by magnesium; maintaining magnesium concentration is a precondition for adenosine triphosphate regeneration following ischemia and reperfusion damage. Magnesium causes vasodilatation by stimulation of endothelial prostacyclin release, the latter prevents vasoconstriction. The NMDA receptor is a voltage-dependent ion channel; magnesium causes increasing extracellular magnesium concentrations by noncompetitive NMDA receptor blockade. Magnesium could protect both hippocampal neurons and white matter, avoid damage by glutamate-mediated necrosis and prolonged ischemia. In animal research, intracellular free magnesium concentration significantly declines after focal fluid-percussion injury in rats, which is associated with the decrease in intracellular high-energy phosphate stores and is in proportion to the severity of injury. Increasing extracellular magnesium concentration improves the recovery of hippocampal neuronal high-energy phosphates and accelerates regional cerebral blood flow to the ischemic brain area after ischemia. ${ }^{21-24}$ As a nonspecific antagonist of all subtypes of voltage-sensitive calcium channels, magnesium ion blocks the NMDA subclass of glutamate receptor and enhances recovery of cellular energy metabolism after ischemia. ${ }^{21-23} \mathrm{In}$ addition, magnesium therapy facilitates cognitive recovery and improves behavioral outcome after brain injury. ${ }^{25,26}$

The benefit of magnesium in therapeutic settings is considered more advantageous than that of many new drugs in recent years, such as safety and tolerability. The ideal dose of magnesium still remains unknown, but the experimental data show that the more the concentration of extracellular magnesium is at the local ischemic site, the greater will be the neuroprotective effect. The therapeutic dose for magnesium should be personalized, serum concentrations of $4-6 \mathrm{mmol} / \mathrm{L}$ are necessary, and in the meanwhile, the symptomatic inhibition of neuromuscular transmission must be monitored and avoided. The goal of this study was to enhance higher physiological concentration of magnesium by injecting extrinsic magnesium and maintain the high concentration levels for 24 hours. The cumulative survival rate (Figures 4 and 5) shows that the therapy of magnesium does not significantly improve the prognosis of patients at discharge, but may improve long-term prognosis.

\section{Conclusion}

In summary, this study was powered to detect differences in clinical outcome, and efficacy was inferred from the results. There was a trend toward favorable outcome in magnesium-treated DAI patients. This is consistent with previous observations. In conclusion, this study showed that magnesium sulfate therapy is effective in the treatment of patients with DAI.

\section{Author contributions}

All authors contributed toward data analysis, drafting and revising the paper and agree to be accountable for all aspects of the work. We would like to thank Christer Ström for assistance with manuscript revision. 


\section{Disclosure}

The authors declare no conflicts of interest involved in this paper.

\section{References}

1. Adams JH, Doyle D, Ford I, Gennarelli TA, Graham DI, McLellan DR. Diffuse axonal injury in head injury: definition, diagnosis and grading. Histopathology. 1989;15(1):49-59.

2. Moen KG, Brezova V, Skandsen T, Håberg AK, Folvik M, Vik A. Traumatic axonal injury: the prognostic value of lesion load in corpus callosum, brain stem, and thalamus in different magnetic resonance imaging sequences. J Neurotrauma. 2014;31(17):1486-1496.

3. Christman CW, Grady MS, Walker SA, Holloway KL, Povlishock JT. Ultrastructural studies of diffuse axonal injury in human. J Neurotrauma. 1994;11(2):173-186.

4. Maqnoni S, Esparza TJ, Conte V, et al. Tau elevations in the brain extracellular space correlate with reduced amyloid- $\beta$ levels and predict adverse clinical outcomes after severe traumatic brain injury. Brain. 2012;135(4):1268-1280.

5. Kara H, Bayir A, Ak A, et al. Trauma in elderly patients evaluated in a hospital emergency department in Konya, Turkey: a retrospective study. Clin Interv Aging. 2014;9:17-21.

6. Gennarelli TA. Animate models of human head injury. JNeurotrauma. 1994;11(4):357-368.

7. Marinov MB, Harbaugh KS, Hoopes PJ, Pikus HJ, Harbaugh RE. Neuroprotective effects of preischemia intraarterial magnesium sulfate in reversible focal cerebral ischemia. J Neurosurg. 1996;85(1): $117-124$.

8. Heath DL, Vink R. Traumatic brain axonal injury produces sustained decline in intracellular free magnesium concentration. Brain Res. 1996; 738(1):150-153.

9. Gee JB 2nd, Corbett RJ, Perlman JM, Garcia D, Laptook AR. Agedependent differences in the relationship between plasma and brain extracellular fluid concentrations of magnesium after $\mathrm{MgSO}_{4}$ infusions in miniswine. Pediatr Res. 1999;46(3):281-286.

10. Vennemeyer JJ, Hopkins T, Kuhlmann J, Heineman WR, Pixley SK. Effects of elevated magnesium and substrate on neuronal numbers and neurite outgrowth of neural stem/progenitor cells in vitro. Neurosci Res. 2014;84:72-78.

11. Wang WP, Dong JJ. A clinical study on hypothermia treatment for severe diffuse axonal injury. Chin J Traumatol. 2002;18:415-417.

12. Marion DW, White MJ. Treatment of experimental brain injury with moderate hypothermia and 21-aminosteriods. J Neurotrauma. 1996;13(3): 139-147.
13. Feldman Z, Gurevitch B, Artru AA, et al. Effect of magnesium given 1 hour after head trauma on brain edema and neurological outcome. J Neurosurg. 1996;85(1):131-137.

14. Mata-Mbemba D, Mugikura S, Nakagawa A, et al. Intraventricular hemorrhage on initial computed tomography as marker of diffuse axonal injury after traumatic brain injury. J Neurotrauma. 2014;32(5): 359-365.

15. Povlishock JT. Traumatically induced axonal injury: pathogenesis and pathobiological implications. Brain Pathol. 1992;2(1):1-12.

16. Weeks D, Sullivan S, Kilbaugh T, Smith C, Margulies SS. Influences of developmental age on the resolution of diffuse traumatic intracranial hemorrhage and axonal injury. J Neurotrauma. 2014;31(2):206-214.

17. McDonald JW, Silverstein FS, Johnston MV. Magnesium reduces N-methyl-D-aspartate (NMDA)-mediated brain injury in perinatal rats. Neurosci Lett. 1990;109(1-2):234-238.

18. Vink R, O'Connor CA, Nimmo AJ, Heath DL. Magnesium attenuates persistent functional deficits following diffuse traumatic brain injury in rats. Neurosci Lett. 2003;336(1):41-44.

19. Heath DL, Vink R. Improved motor outcome in response to magnesium therapy received up to 24 hour after traumatic diffuse axonal brain injury in rats. J Neurosurg. 1999;90(3):504-509.

20. Rowe WJ. Correcting magnesium deficiencies may prolong life. Clin Interv Aging. 2012;7:51-54.

21. Clerc P, Young CA, Bordt EA, Grigore AM, Fiskum G, Polster BM. Magnesium sulfate protects against the bioenergetic consequences of chronic glutamate receptor stimulation. PLoS One. 2013;8(11):e79982.

22. Altura BM, Barbour RL, Dowd TL, Wu F, Altura BT, Gupta RK. Low extracellular magnesium induces intracellular free $\mathrm{Mg}$ deficits, ischemia, depletion of high-energy phosphates and cardiac failure in intact working rat hearts: a 31P-NMR study. Biochim Biophys Acta. 1993;1182(3):329-332.

23. Stys PK, Ransom BR, Waxman SG. Effects of polyvalent cations and dihydropyridine calcium channel blockers on recovery of CNS white matter from anoxia. Neurosci Lett. 1990;115(2-3):293-299.

24. Muir KW, Lees KR. Dose optimization of intravenous magnesium sulfate after acute stroke. Stroke. 1998;29(5):918-923.

25. Hoane MR. Assessment of cognitive function following magnesium therapy in the traumatically injured. Magnes Res. 2007;20(4):229-236.

26. Hoane MR. Treatment with magnesium improves reference memory but not working memory while reducing GFAP expression following traumatic brain injury. Restor Neurol Neurosci. 2005;23(2):67-77.
Therapeutics and Clinical Risk Management

\section{Publish your work in this journal}

Therapeutics and Clinical Risk Management is an international, peerreviewed journal of clinical therapeutics and risk management, focusing on concise rapid reporting of clinical studies in all therapeutic areas, outcomes, safety, and programs for the effective, safe, and sustained use of medicines. This journal is indexed on PubMed Central, CAS,
Dovepress

EMBase, Scopus and the Elsevier Bibliographic databases. The manuscript management system is completely online and includes a very quick and fair peer-review system, which is all easy to use. Visit $\mathrm{http}: / / \mathrm{www}$.dovepress.com/testimonials.php to read real quotes from published authors. 